\title{
ariacar

\section{Generation of X-points and secondary islands in 2D magnetohydrodynamic turbulence}

Minping Wan, William H. Matthaeus, Sergio Servidio, and Sean Oughton

Citation: Phys. Plasmas 20, 042307 (2013); doi: 10.1063/1.4802985

View online: http://dx.doi.org/10.1063/1.4802985

View Table of Contents: http://pop.aip.org/resource/1/PHPAEN/v20/i4

Published by the American Institute of Physics.

\section{Additional information on Phys. Plasmas}

Journal Homepage: http://pop.aip.org/

Journal Information: http://pop.aip.org/about/about_the_journal

Top downloads: http://pop.aip.org/features/most_downloaded

Information for Authors: http://pop.aip.org/authors

\section{ADVERTISEMENT}

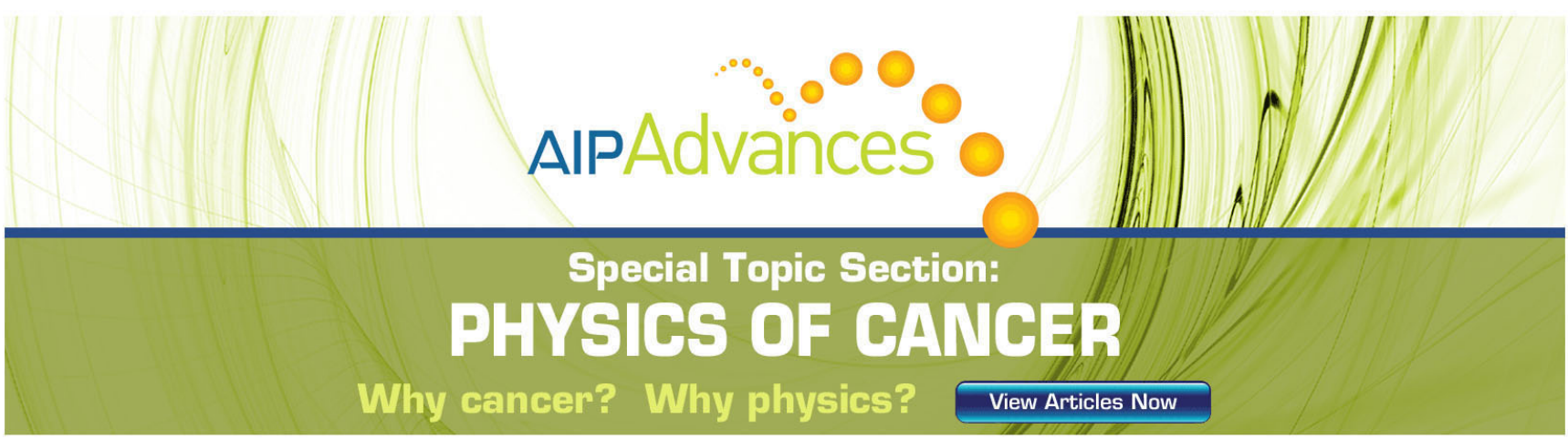




\title{
Generation of X-points and secondary islands in 2D magnetohydrodynamic turbulence
}

\author{
Minping Wan, ${ }^{1}$ William H. Matthaeus, ${ }^{1}$ Sergio Servidio, ${ }^{2}$ and Sean Oughton ${ }^{3}$ \\ ${ }^{1}$ Bartol Research Institute and Department of Physics and Astronomy, University of Delaware, Newark, \\ Delaware 19716, USA \\ ${ }^{2}$ Dipartimento di Fisica, Universita' della Calabria, I-87036 Cosenza, Italy \\ ${ }^{3}$ Department of Mathematics, University of Waikato, Hamilton 3240, New Zealand
}

(Received 18 March 2013; accepted 12 April 2013; published online 29 April 2013)

\begin{abstract}
We study the time development of the population of X-type critical points in a two-dimensional magnetohydrodynamic model during the early stages of freely decaying turbulence. At sufficiently high magnetic Reynolds number $R e_{m}$, we find that the number of neutral points increases as $R e_{m}^{3 / 2}$, while the rates of reconnection at the most active sites decrease. The distribution of rates remains approximately exponential. We focus in particular on delicate issues of accuracy, which arise in these numerical experiments, in that the proliferation of X-points is also a feature of under-resolved simulations. The "splitting" of neutral points at high Reynolds number appears to be a fundamental feature of the cascade that has important implications for understanding the relationship between reconnection and turbulence, an issue of considerable importance for the Magnetospheric Multiscale and Solar Probe missions as well as observation of reconnection in the solar wind. (C) 2013 AIP Publishing LLC. [http://dx.doi.org/10.1063/1.4802985]
\end{abstract}

\section{INTRODUCTION}

Magnetic reconnection in two-dimensional (2D) magnetohydrodynamics (MHD) is mediated by activity near magnetic X-type neutral points, and plays an important role in the turbulent cascade. ${ }^{1-3}$ In three dimensions, reconnection is still more complex ${ }^{4}$ and becomes essential, for example, in the dynamo process. ${ }^{5}$ Owing to this complexity, and the associated demands on computational resources at high Reynolds numbers, the influence of turbulence on the reconnection process has mainly been investigated in the $2 \mathrm{D}$ case. Furthermore, the 2D case remains of interest when 3D turbulence is anisotropic and becomes "quasi-two dimensional." "6-8 One of the distinctive turbulence effects in reconnection is the appearance of multiple X-points, which are potentially associated with both particle energization ${ }^{9}$ and rapid reconnection ${ }^{10}$ Recently, there has been an increase in activity in this area, including study of "plasmoid instability,"11-14 in simulation of directly driven turbulent reconnection activity, ${ }^{15}$ and analysis of the statistics of reconnection rate in snapshots of $2 \mathrm{D}$ turbulence. ${ }^{16-18}$ These relationships have prompted various unified perspectives on turbulence and reconnection. ${ }^{1-3,18-20}$ Here, we continue this inquiry by further examining the emergence and role of secondary islands in 2D MHD turbulence. We focus on issues related to the number of islands and magnetic X-points in well-resolved spectral method simulations, as the Reynolds numbers are increased. We find that the number of magnetic islands generally increases in time when initial conditions are band-limited, while moving towards higher Reynolds numbers using a fixed class of initial data generally leads to larger numbers of X-points and secondary islands.

At a fundamental level, reconnection involves change of magnetic connectivity and topology, along with release of magnetic energy into heat, flows and in a plasma, suprathermal particles. ${ }^{21,22}$ In $2 \mathrm{D}$, the topology is that of flux tubes or "islands" (sometimes also called "bubbles" or "plasmoids") and the lanes between them, and so the level of complexity is related to the number of $\mathrm{X}$ - or O-type neutral points in the in-plane magnetic field. In turbulence, flux tubes and vortices constantly interact, producing stretching, merging, and a cascade across scale. In such an environment, with constantly changing connectivity and topology, it is not surprising that the size and number of islands (and X-points) will also change. We quantify this effect here through identification of $\mathrm{X}$-points in evolving 2D MHD turbulence, and characterization of the statistics of the X-type critical points.

We remark briefly that it remains desirable but very difficult to carry out this type of study in three dimensions. Unfortunately, computational limitations are prohibitive for the required high resolution, high Reynolds number simulations. Similarly, the required high resolution at MHD scales needed to see the effects we describe, appear to remain beyond what is attainable using fully kinetic plasma simulation (see, however, Refs. 14, 23, and 24). These extensions may be especially important with regard to applications in space, laboratory, and astrophysical settings. However, in order to focus on properties of reconnection and turbulence occurring in a "large" system possessing a wide range of dynamically involved spatial scales, we choose to avoid these additional demanding extensions. We focus here on the emergence of broad band cascade, intermittency, and dynamical complexity. These effects, as well as plasmoid instability, emerge when Reynolds numbers are large. To attain these conditions and assure adequate numerical accuracy, we use a 2D MHD model throughout the present study.

\section{NUMERICAL SIMULATIONS AND METHOD}

Our computations solve the 2D incompressible MHD equations expressed in terms of the vector potential $a(x, y, t)$ and vorticity $\omega=(\nabla \times \boldsymbol{v}) \cdot \hat{z}$, 


$$
\begin{aligned}
\partial_{t} \omega & =-\nabla \cdot(\omega \boldsymbol{v}-j \boldsymbol{b})+\nu \nabla^{2} \omega, \\
\partial_{t} a & =-\nabla \cdot(a \boldsymbol{v})+\eta \nabla^{2} a,
\end{aligned}
$$

involving the magnetic field $\boldsymbol{b}=\nabla \boldsymbol{a} \times \hat{\boldsymbol{z}}$, electric current density $j=-\nabla^{2} a$, velocity $\boldsymbol{v}$, and viscous and resistive dissipation coefficients $\nu$ and $\eta$.

Equations (1) are solved numerically in a $2 \pi$-periodic square box using a Fourier spectral method with dealiasing via a $2 \sqrt{2} / 3$-rule and a phase-shift technique..$^{25,26}$ Thus, for a resolution of $N \times N$, the maximum retained wavenumber (in each direction) is $k_{\max }=2 \sqrt{2} / 3$. Time is advanced using a second-order Runge-Kutta method. The initial $(t=0)$ spectra of $\boldsymbol{v}$ and $\boldsymbol{b}$ are chosen proportional to $\left[1+\left(\frac{k}{k_{0}}\right)^{8 / 3}\right]^{-1}$, within a band of $k$, and the phases of the Fourier coefficients are assigned using Gaussian random numbers; $k=|\boldsymbol{k}|$ is the magnitude of the Fourier wavevector. The initial kinetic and magnetic energies are equal, $E_{v}=\left\langle|\boldsymbol{v}|^{2}\right\rangle / 2=0.5, E_{b}$ $=\left\langle|\boldsymbol{b}|^{2}\right\rangle / 2=0.5$. The cross helicity $H_{c}=\langle\boldsymbol{v} \cdot \boldsymbol{b}\rangle$ is small initially and remains so during the runs. Angle brackets $\langle\cdots\rangle$ denote a volume average over the box.

Since $\nu=\eta$ herein, the dissipation wavenumber (reciprocal of the Kolmogorov scale $\left.\lambda_{\mathrm{d}}\right)^{29}$ is defined as

$$
k_{\mathrm{diss}}(t)=\frac{1}{\lambda_{\mathrm{d}}}=\left(\frac{\epsilon}{\nu^{3}}\right)^{1 / 4} \equiv \frac{\left\langle\omega^{2}+j^{2}\right\rangle^{1 / 4}}{\sqrt{\nu}},
$$

where $\langle\cdots\rangle$ denotes spatial averaging and $\epsilon$ is the average rate of energy dissipation. The ratio $r(t) \equiv k_{\max } / k_{\mathrm{diss}}(t)$ $\equiv k_{\max } \lambda_{\mathrm{d}}$ can be interpreted as a measure of adequate resolution of the dissipation scale, with larger values indicating better resolution.

Various simulations were performed for a range of resolutions, $k_{0}$ values, and initially excited $k$-bands. Here we report on three series of runs (see Tables I, II, and III).

When the turbulence is fully developed, coherent structures appear. They can be identified as magnetic islands that have different size and energy. In the regions between islands the perpendicular (out-of-plane) component of the current density $j$ may become very high. This is related to the intermittent nature of the magnetic field and can be interpreted as a consequence of fast, local relaxation processes. ${ }^{27}$

The (out-of-plane component of the) magnetic potential $a$ shows a collection of magnetic islands having a wide

TABLE I. Parameters for simulation set 1 , which all have $\nu=\mu=1 / 2000$. The initially excited Fourier modes have $5 \leq|\boldsymbol{k}| \leq 30$ with $k_{0}=10 . k_{\text {diss }}$ means the maximum value of $k_{\text {diss }}(t)$ and $t_{\text {peak }}$ is the time at which it occurs. Runs $1-3$ are definitely under-resolved.

\begin{tabular}{lccrccr}
\hline \hline Run & Grid & $1 / \nu, 1 / \eta$ & $k_{\max }$ & $k_{\text {diss }}$ & $\frac{k_{\max }}{k_{\text {diss }}}$ & X-points \\
\hline A1 & $512^{2}$ & 2000 & 241 & 250 & 0.96 & 1210 \\
A2 & $1024^{2}$ & 2000 & 482 & 251 & 1.9 & 601 \\
A3 & $1536^{2}$ & 2000 & 724 & 251 & 2.9 & 571 \\
A4 & $2048^{2}$ & 2000 & 965 & 251 & 3.9 & 567 \\
A5 & $4096^{2}$ & 2000 & 1930 & 251 & 7.7 & 567 \\
\hline \hline
\end{tabular}

TABLE II. Parameters for the second set of simulations with $k_{0}=15$ and initial $k$-band $[8,40]$.

\begin{tabular}{lccccc}
\hline \hline Run & Grid & $1 / \nu, 1 / \eta$ & $t_{\text {peak }}$ & $\frac{k_{\text {max }}}{k_{\text {diss }}}$ & X-points \\
\hline Run 1 & $2048^{2}$ & 2222 & 0.14 & 3.1 & 963 \\
Run 2 & $4096^{2}$ & 6667 & 0.20 & 3.0 & 1279 \\
Run 3 & $8192^{2}$ & 18182 & 0.30 & 3.0 & 2971 \\
Run 4 & $16384^{2}$ & 45455 & 0.35 & 3.1 & 7945 \\
\hline \hline
\end{tabular}

TABLE III. Parameters for the third set of simulations with $k_{0}=10$ and initial $k$-band $[5,20]$.

\begin{tabular}{lrrrrr}
\hline \hline Run & Grid & $1 / \nu, 1 / \eta$ & $t_{\text {peak }}$ & $\frac{k_{\text {max }}}{k_{\text {diss }}}$ & X-points \\
\hline Run 5 & $2048^{2}$ & 2857 & 0.38 & 3.2 & 373 \\
Run 6 & $4096^{2}$ & 8333 & 0.48 & 3.1 & 709 \\
Run 6a & $8192^{2}$ & 8333 & 0.48 & 6.1 & 686 \\
Run 7 & $8192^{2}$ & 20000 & 0.54 & 3.3 & 1487 \\
Run 7a & $16384^{2}$ & 20000 & 0.54 & 6.6 & 1435 \\
Run 8 & $16384^{2}$ & 50000 & 0.80 & 3.4 & 5649 \\
\hline \hline
\end{tabular}

distribution of sizes. In three dimensions these would correspond to flux tubes. For the large islands, the sign of $a$ in a closed field-line region gives the sense of rotation of the magnetic vortex. These coherent structures interact nonlinearly, merge, stretch, attract, and repulse each other. In fact, the dynamics of the magnetic field in 2D MHD turbulence can be thought of as consisting largely of the interactions among these islands. Reconnection is a major element of this interaction.

\section{RECONNECTION AND CRITICAL POINTS IN 2D MHD TURBULENCE}

To understand the magnetic reconnection in the 2D turbulent system, we need to examine the topography of the magnetic potential $a(x, y)$ in detail. In particular, we need to identify the neutral points- points where $\nabla a=0$ —and their nature. A useful tool in this regard is the Hessian matrix of $a$, defined as

$$
H_{i j}^{a}(x)=\frac{\partial^{2} a}{\partial x_{i} \partial x_{j}}
$$

At each neutral point, we calculate the eigenvalues of $H_{i j}^{a}(x)$. If both eigenvalues are positive (negative), the point is a local minimum (maximum) of $a$, with both cases generically referred to as O-points. If the eigenvalues are of mixed sign, it is a saddle point, also termed an X-point.

Because of the complex topology of turbulence, critical points can be very close to each other. Moreover, based on a spectral representation, they are usually not located on the vertices of a chosen computational grid, and so we use a second-order interpolation algorithm to determine their positions. However, the presence of energy at the smallest scales affects the precision of the interpolation technique, producing false critical points.

To avoid this problem, we interpolate all analyzed realspace fields onto a $32768^{2}$ grid. This is achieved using zero- 
padding in an expanded Fourier space as we now describe in more detail. First, the Fourier transform of $a(x, y)$ is computed, yielding the Fourier coefficients $\hat{a}\left(k_{x}, k_{y}\right)$. These are copied into the bigger $32768^{2}$ array in such a way that the expanded array has identical Fourier amplitudes at wavevectors that were present in the lower resolution representation. The remaining modes are set to zero, i.e., those with $k_{j} \geq N / 2$, where $N / 2$ is the maximum wavevector component of the original array (Nyquist frequency). Following this zero-padding, we inverse Fourier transform to obtain $a(x, y)$ on a higher resolution spatial grid. In this way we can generate a function that has $32768 \times 32768$ points from the original $N \times N$ points. It coincides with the original function on the original grid points. Between these, on the new finer grid, it represents a trigonometric interpolation of the function. Although this process can require substantial computer memory, it gives results with the following desirable properties: (1) a function extrapolated onto a higher resolution grid with an exact Fourier expansion, (2) cases in which critical points are in the same Cartesian cell are avoided, and (3) the interpolation becomes much more accurate, even if the order of the interpolation is the same (this is because the field is much smoother at the new grid size).

\section{A STUDY OF THE QUALITY OF RESOLUTION}

The accuracy of interpolation is not the only factor that impacts the quality of the results when studying critical points. Spatial resolution of the numerical method is of even greater importance in computing the dynamics from which the coherent structures' critical points emerge. To study this, we first consider a series of runs (Table I) in which the initial physical parameters-including the Reynolds numbers-are fixed, and the numerical resolution is varied. For more details of these runs, see Refs. 28.

Energy spectra for the runs listed in Table I, are displayed in Fig. 1. Clearly all the spectra agree well. Indeed, although the spectra cut off at different wavenumbers (because of the different run resolutions), the curves nearly overlay each other over the full range of overlapping $k$, with only small discrepancies in the lower resolution runs near their maximum retained wavenumber.

In Fig. 2 we show the number of X-points found as a function of time for all runs in Table I. We observe that the

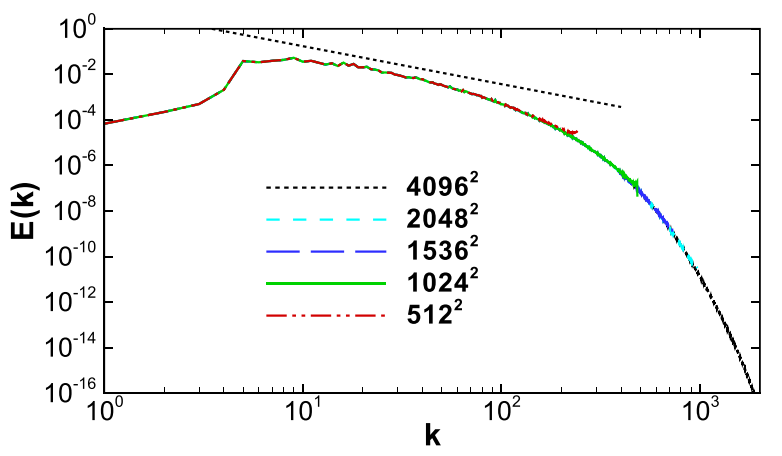

FIG. 1. Energy spectra at the time of maximum $\left\langle j^{2}\right\rangle, t_{\text {peak }}$, for all runs in simulation set 1 in Table I (fixed $\nu$, varying resolution). A $k^{-5 / 3}$ power-law (dotted) is shown for comparison.

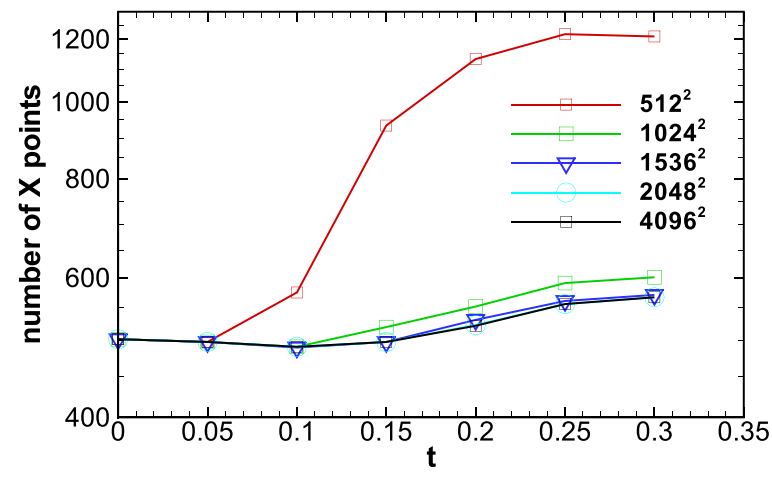

FIG. 2. Number of X-points found as a function of time for all runs in Table I.

under-resolved runs, e.g., the $512^{2}$ run, generate many more $\mathrm{X}$-points than the well-resolved runs. However, the number of X-points converges as the resolution increases. As reported in Ref. 28 , it is required to have

$$
\frac{k_{\max }}{k_{\mathrm{diss}}} \equiv k_{\max } \lambda_{\mathrm{d}}>3,
$$

to obtain an accurate number of X-points [see Eq. (2)].

\section{RECONNECTION AT HIGH REYNOLDS NUMBER}

To perform the statistical analysis of X-point features, we consider the state of each system at the time $\left(t_{\text {peak }}\right)$ when the mean-square current density $\left\langle j^{2}\right\rangle$ reaches its peak value. At this time the peak of small-scale turbulent activity is achieved. For orientation, Fig. 3 displays the familiar omnidirectional power spectra of the magnetic field $E_{b}(k)$ at those
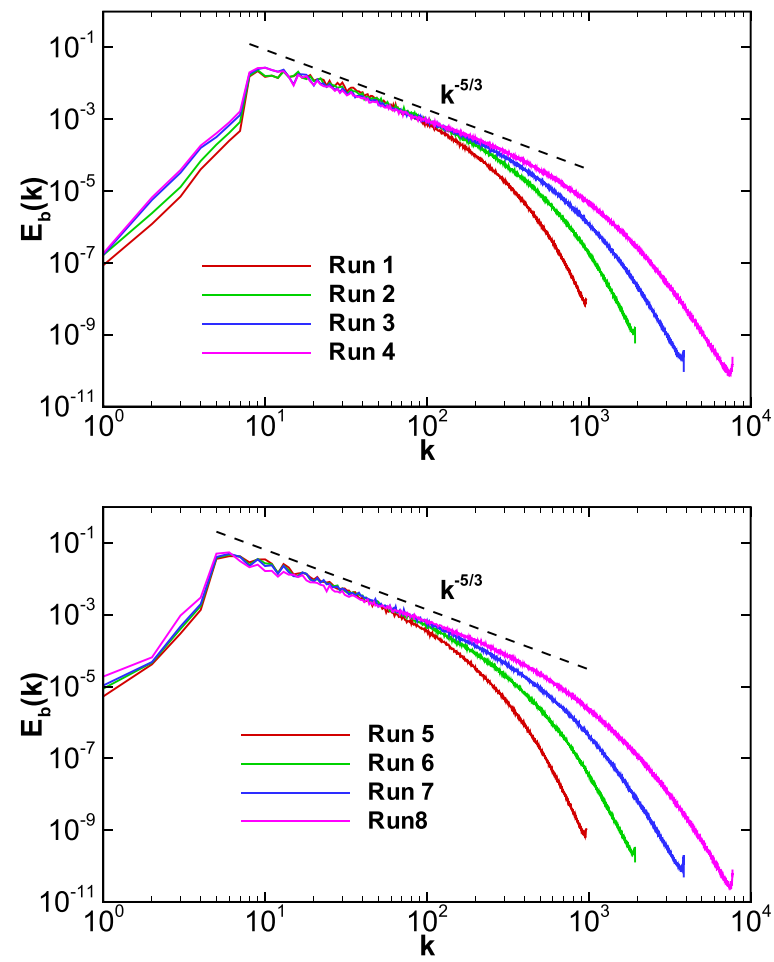

FIG. 3. Magnetic energy spectra at $t=t_{\text {peak }}$ for Table II runs (top) and Table III runs (bottom). 
times, for some simulations listed in Tables II and III. An inertial range is usually evident.

Using the procedure of Sec. III, we determined the X-point sites for all runs in Tables II and III (at $t=t_{\text {peak }}$ ). The number of potential reconnection sites is listed in these tables. Note that each of these tables describes a sequence of runs having increasing Reynolds number, for a fixed set of initial conditions. Every run in these two sequences satisfies the empirical accuracy requirement $k_{\max }>3 k_{\text {diss }}$ on the dissipation wavenumber $k_{\text {diss }}$ and the maximum resolved wavenumber $k_{\max }$ that is discussed above. With this confidence in the accuracy established, we may then compare the number of X-points found for a particular run (see Tables II and III), with the magnetic energy spectrum plotted in Fig. 3. It is apparent that there are many more X-points found for the runs with larger Reynolds number, especially for two $16384^{2}$ runs. With their energy spectrum almost the same at lower wavenumbers, and all runs fully developed, the observed increase of X-points should be associated with the much smaller scales present in the higher Reynolds number, higher resolution runs. These smaller scales are not dynamically accessible in the lower Reynolds number runs.

Having obtained the X-point sites, we may then compute the reconnection rates as (minus) the electric field at the $\mathrm{X}$-point, or equivalently the rate of change of the magnetic flux in the strong field regions adjoining the $\mathrm{X}$-point,

$$
\frac{\partial a}{\partial t}=(\eta j)_{X}=-E_{X}
$$

Here $E_{X}$ is an abbreviation for the electric field measured at the (X-point) saddle point.

Figure 4 shows the probability density functions (PDFs) of the magnetic reconnection rates computed from Table II runs. They are all quite broad and peaked around the zero value. The lower panel shows the PDFs of the absolute values of these reconnection rates. Similar results are obtained for the Table III runs (not shown).

It is easy to observe that generally the value of the reconnection rate becomes smaller for larger Reynolds number simulations. To better understand their statistics, we also plot the PDFs of the magnetic reconnection rates normalized by their corresponding rms values, designated as $\sigma$ (Fig. 5). We observe that the normalized PDFs collapse to each other, and that the tails generally extend further for larger Reynolds numbers. The same behavior is also observed in the normalized PDFs of the absolute values of reconnection rate, shown in the lower panel. It is evident that the distribution of reconnection rates behaves as $P\left(E_{X}\right) \sim e^{-\left|E_{X}\right| / \sigma}$ to a reasonable degree of accuracy over the accessible range of reconnection rates.

The distribution of reconnection rates can be understood as follows. We know that at an X-point the reconnection rate is $E_{X}=\eta J$, and that the total (magnetic) dissipation $\left\langle\eta J^{2}\right\rangle$ is approximately independent of Reynolds number for large Reynolds number turbulence. ${ }^{30}$ Thus, $\left\langle E_{X}^{2}\right\rangle=\left\langle\eta^{2} J^{2}\right\rangle \sim \eta \sim$ $1 / R e_{m}$ will decrease as $\eta$ decreases. Note the bracket in $\left\langle E_{X}^{2}\right\rangle$ is an average on the X-points, while the second bracket $\left\langle\eta^{2} J^{2}\right\rangle$ is a global average in space. The idea is that the
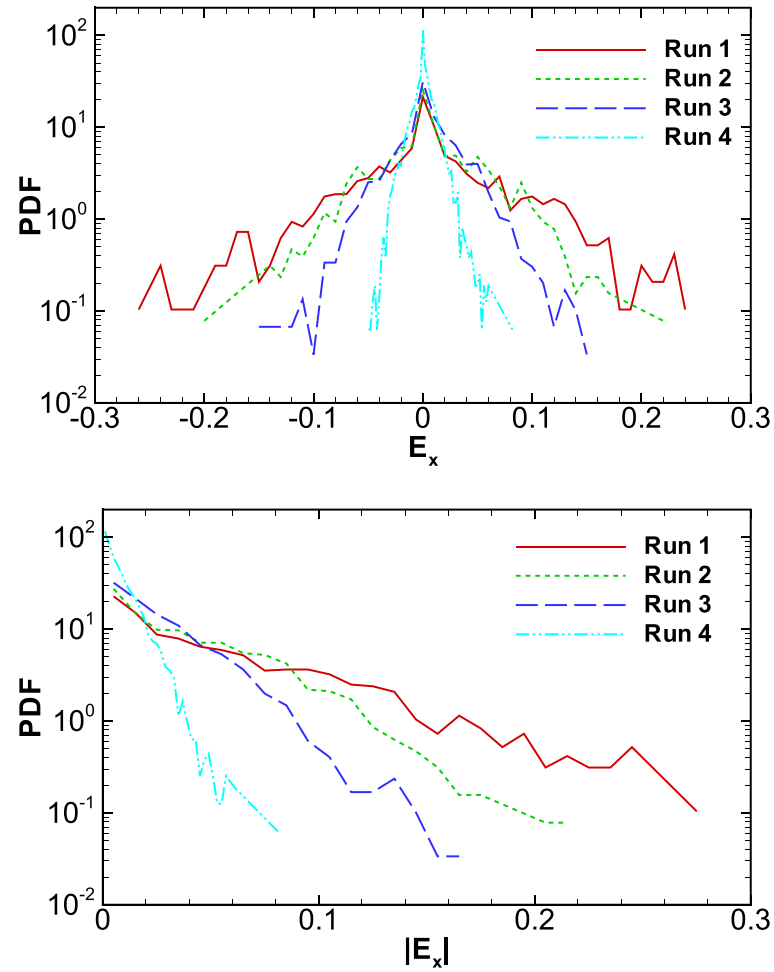

FIG. 4. PDFs of the magnetic reconnection rates (top) and their absolute values (bottom) from Table I runs at the time of maximum $\left\langle j^{2}\right\rangle$.

dissipation at X-points scales like the global dissipation. In this simple analysis, the rms reconnection rate would scale as $\sim R e_{m}^{-1 / 2}$. To empirically quantify this, we plot in Fig. 6 the rms value of the magnetic reconnection rates $\sigma=E_{\mathrm{rms}}^{X}$ as
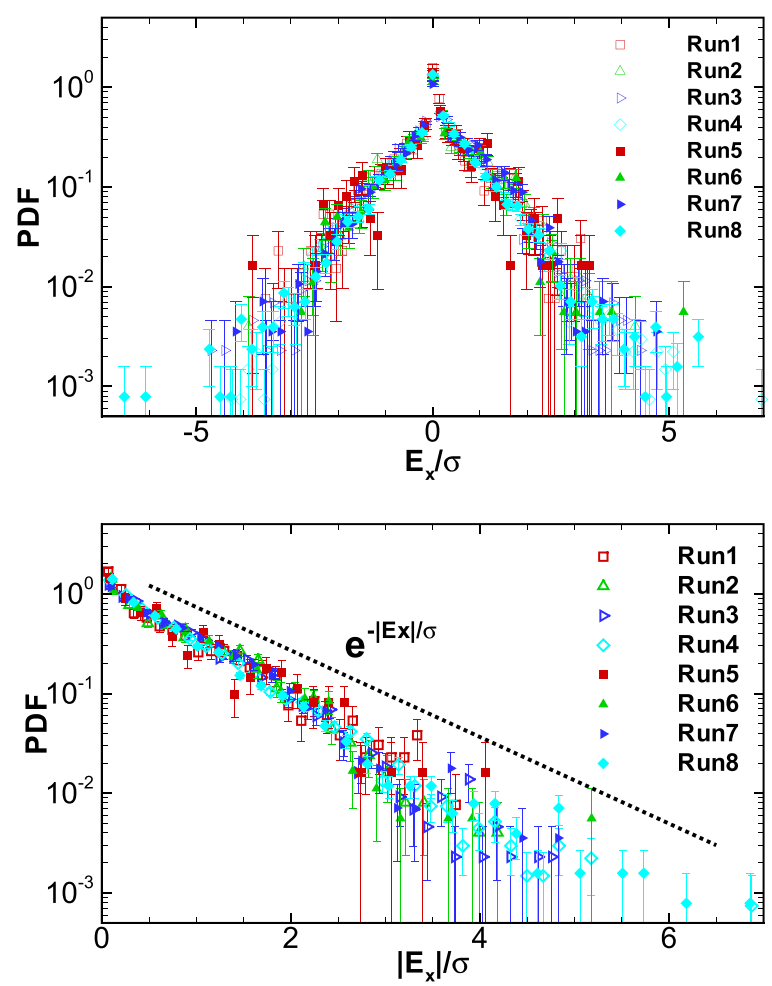

FIG. 5. PDFs of the magnetic reconnection rates (top) and their absolute values (bottom), each normalized by their rms values $\sigma=E_{r m s}^{X}$. Data are from Tables II and III runs at the time of maximum $\left\langle j^{2}\right\rangle$. 


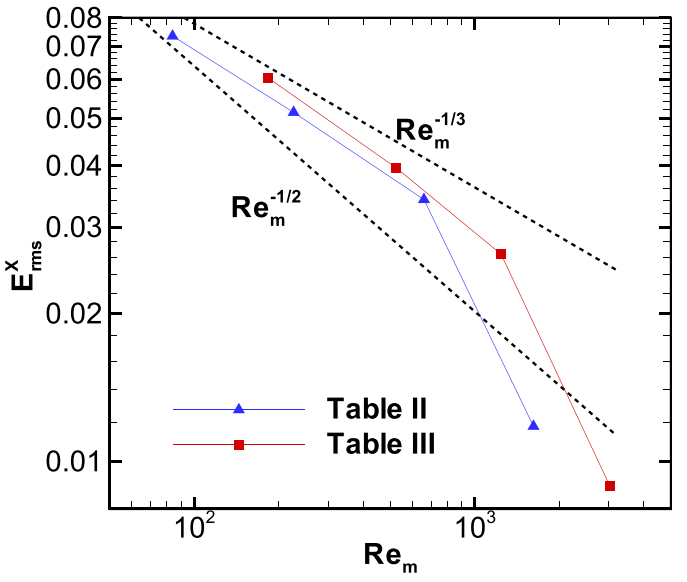

FIG. 6. RMS value of the magnetic reconnection rates as a function of the magnetic Reynolds number.

a function of $R e_{m}$. The magnetic Reynolds numbers are calculated with the correlation length $L_{b}$ of the magnetic field, defined as

$$
L_{b}=\frac{2}{b_{\mathrm{rms}}^{2}} \int \frac{E_{b}(k)}{k} \mathrm{~d} k
$$

where $b_{\mathrm{rms}}=\sqrt{\left\langle b_{x}^{2}+b_{y}^{2}\right\rangle}$ is the rms magnetic field strength. For both sets of runs, we observe some range with scaling between $E_{\mathrm{rms}}^{X} \sim R e_{m}^{-1 / 3}$ and $E_{\mathrm{rms}}^{X} \sim R e_{m}^{-1 / 2}$, except for the two largest $16384^{2}$ runs.

As our results show, the number of X-points, and therefore potential reconnection sites, increases dramatically with Reynolds number. To make this more quantitative, we study the density of X-points $\rho_{X}$, defined as the (average) number of X-points per square box of size $L_{a} \times L_{a},{ }^{31,32}$ where $L_{a}$ is the correlation length of the magnetic potential $a$, calculated using an equation similar to Eq. (6). Figure 7 plots $\rho_{X}$ as a function of magnetic Reynolds number for Tables II and III runs, which differ in their initial data. Once again we employ results from the time of peak $\left\langle j^{2}\right\rangle$. It is evident that the spatial density of reconnection sites increases with Reynolds number in a very similar way for the two families of runs.

To eliminate the effect of the initial field in this analysis, we calculate the increase in the density of X-points, defined as $\rho_{X}\left(t_{\text {peak }}\right)-\rho_{X}(t=0)$, shown in the right panel of Fig. 7. This quantity is due entirely to the development of the cascade and is expected to be most relevant when the initial
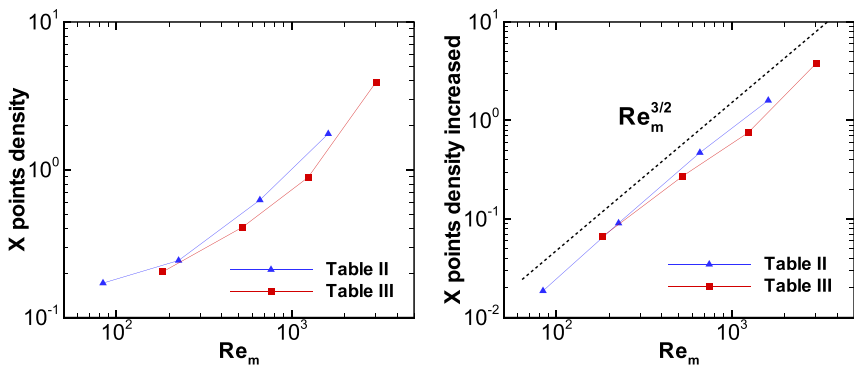

FIG. 7. Left: Density of X-points as a function of the magnetic Reynolds number. Right: Increased density of X-points as a function of the magnetic Reynolds number. populated $\boldsymbol{k}$-space is of relatively narrow bandwidth in wavenumber. As the figure shows, the increase in density of $\mathrm{X}$-points follows an almost perfect $R e_{m}^{3 / 2}$ scaling. This may also be interpreted as the increase in density of dynamically produced secondary islands or "plasmoids." A simple line of reasoning serves to account for this scaling: As discussed above, the emergence of new X-points and secondary islands is connected with the development of the nonlinear cascade, as the system approaches a fully developed state. During this development, the cascade eventually extends to and declines sharply beyond the Kolmogorov dissipation wavenumber $k_{\text {diss }}=1 / \lambda_{\mathrm{d}}$. We argue that the increase in number of $\mathrm{X}$-points should scale as the volume of wavevector space that becomes populated by the cascade, which is $\sim k_{\text {diss }}^{2}-k_{0}^{2}$, where $k_{0}$ is some measure of the bandwidth of the initial data. (Note that $k_{0}^{2}$ is a measure of number of initial islands.) At high Reynolds number, the first contribution greatly exceeds the second. Recalling, for example, from Eq. (2), that $k_{\text {diss }} \sim R e_{m}^{3 / 4}$, one finds that the expected increase in the number of $\mathrm{X}$-points $\sim k_{\text {diss }}^{2} \sim R e_{m}^{3 / 2}$, which agrees very well with the scaling seen in Fig. 7.

\section{TIME EVOLUTION OF MAGNETIC RECONNECTION}

In this section, we will discuss the time-dependent statistics of the number of $\mathrm{X}$-points and of the rates of magnetic reconnection.

In Fig. 8, we show the number of X-points found as a function of time for Runs 6 and 7. We observe that the number of X-points increases for both runs. The accuracy of the increases is confirmed by Runs $6 \mathrm{a}$ and $7 \mathrm{a}$, which have initial fields and physical parameters that are exactly the same as Runs 6 and 7, but have their resolutions doubled, respectively. The results from Runs 6 and $6 a$, and Runs 7 and $7 a$ are found to be nearly identical, which confirms that the increasing of the number of X-points seen in comparing Runs 6 and 7 is of physical origin, and is not caused by inadequate spatial resolution.

We also observe that the number of X-points is larger (and increases faster) for Run 7 than Run 6, which further demonstrates that Reynolds number plays a role in the increasing of the number of X-points, as discussed in Sec. V.

An additional point of emphasis is that there remains a slight $(3 \%$ or $4 \%)$ decrease in number of X-points in going

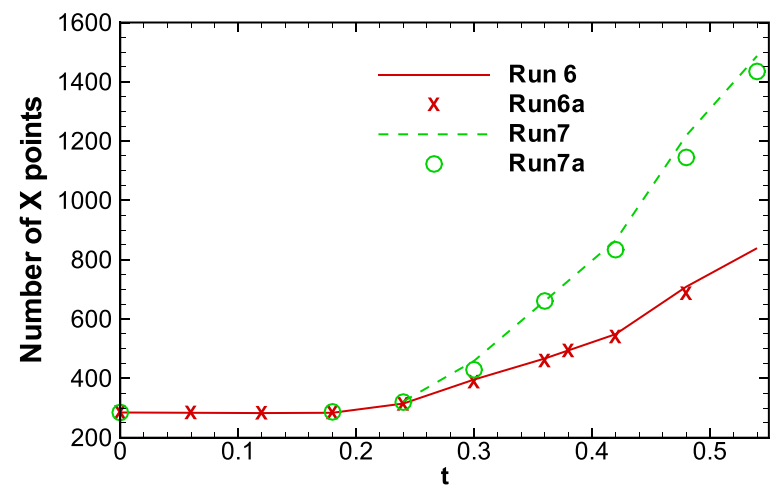

FIG. 8. Number of X-points found as a function of time for four of the runs in Table III. 
from Runs 6 to $6 \mathrm{a}$, and in going from Runs 7 to $7 \mathrm{a}$. While this decrease is small, it is not completely negligible. The suggestion can be made that the resolution of these runs, even at $k_{\max } \lambda_{\mathrm{d}}>3$, may not be quite as good in attaining convergence as it was at lower Reynolds numbers. It is possible that at higher $R e_{m}$ even greater resolution relative to the Kolmogorov scale will be required.

To further demonstrate the role of Reynolds number, in Fig. 9 we display the energy spectrum at four different times for the highest Reynolds number Run 8. In the same figure, we also plot the number of X-points found as a function of time from the same run. We can see that in this run, as the spectrum fills out by transfer of greater amounts of energy to higher wavenumber, one sees a concomitant increase in the number of X-points, from less than 300 to more than 5000.

Another feature of interest is the dependence of $E_{X}$ on the local geometry of the diffusion region near each $\mathrm{X}$-point. This is related to the eigenvalues of the Hessian matrix, ${ }^{16,17}$

$$
\lambda_{\max }=\frac{\partial^{2} a}{\partial s^{2}}, \quad \lambda_{\min }=\frac{\partial^{2} a}{\partial l^{2}},
$$

the larger and smaller (in magnitude), respectively, and the associated unit eigenvectors $\hat{\boldsymbol{e}}_{s}$ and $\hat{\boldsymbol{e}}_{l}$. The coordinate $s$ may be associated with the minimum thickness $\delta$ of the current sheet, and $l$ with the elongation $\ell$. From a scaling analysis of Eq. (7), the aspect ratio of the diffusion region is well approximated by

$$
\frac{\ell}{\delta} \approx \sqrt{\lambda_{R}}, \text { where } \lambda_{R}=\left|\frac{\lambda_{\max }}{\lambda_{\min }}\right| .
$$
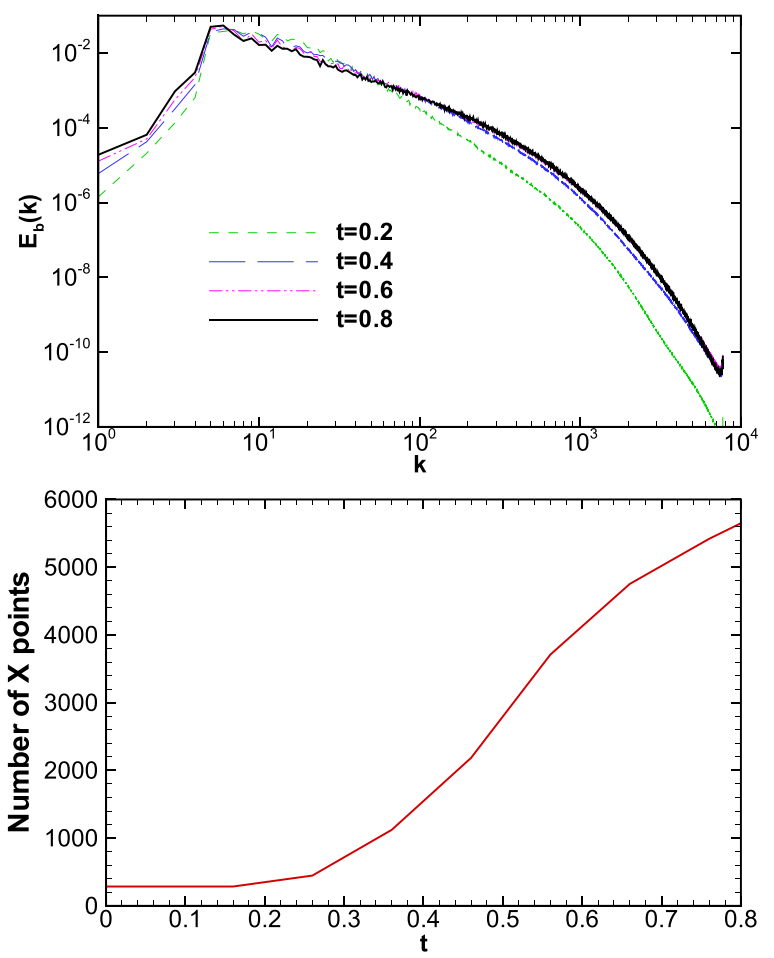

FIG. 9. Top: Evolution of the magnetic energy spectrum for Run 8. Bottom: Number of X-points found as a function of time.
In Fig. 10, we illustrate the relationship between the reconnection rate (electric field) at each X-point, and the nominal aspect ratio of the reconnection region measured as the ratio of the eigenvalues. These distributions are shown at different times for Run 8. Since the simulation started from Gaussian fields with random phases, at the initial time $t=0$ the reconnection rates are small and no obvious scaling between $\left|E_{X}\right|$ and $\lambda_{R}$ is found. The values of $\lambda_{R}$ are also generally not greatly different from unity. However, at $t=0.22$, we observe that the reconnection rates are generally much larger than the initial values, while the eigenvalue ratio is distributed over a much wider range of values. Furthermore, a clear power-law scaling consistent with Eq. (8) starts to emerge, which is also consistent with the picture that nonGaussian features emerge rapidly in turbulence. ${ }^{33}$ At $t=0.8$ when the turbulence is fully developed, not only the number of reconnection sites has greatly increased but also the associated reconnection rates have also generally increased, following the scaling with $\lambda_{R}$ reported previously. ${ }^{16,17}$

Finally, we illustrate the real space development of secondary islands and emergent $\mathrm{X}$-points by examining the magnetic field-lines and $\mathrm{X}$-point positions in a subregion at several different times. Figure 11 is an example from Run 8, displaying the magnetic configuration in a small region at four times. Comparing the figure panels, we observe that much finer structures ("secondary islands") are formed at later times, which contributes to the dramatic increase of the total number of X-points. There is a suggestion (not completely apparent in the figure due to superposition of symbols) that many of the new X-points are born by "splitting" of an X-point already present. The dynamical stresses that produce this effect must include a non-uniformity of dissipation in the immediate neighborhood. Another example of the proliferation of the number of X-points in a region of highly stressed magnetic field is shown in Fig. 12. In the first panel, at $t=0.36$, there are only two X-points, but it is clear that within this region, several magnetic structures are commencing a complex interaction. Later, at $t=0.46$ this interaction

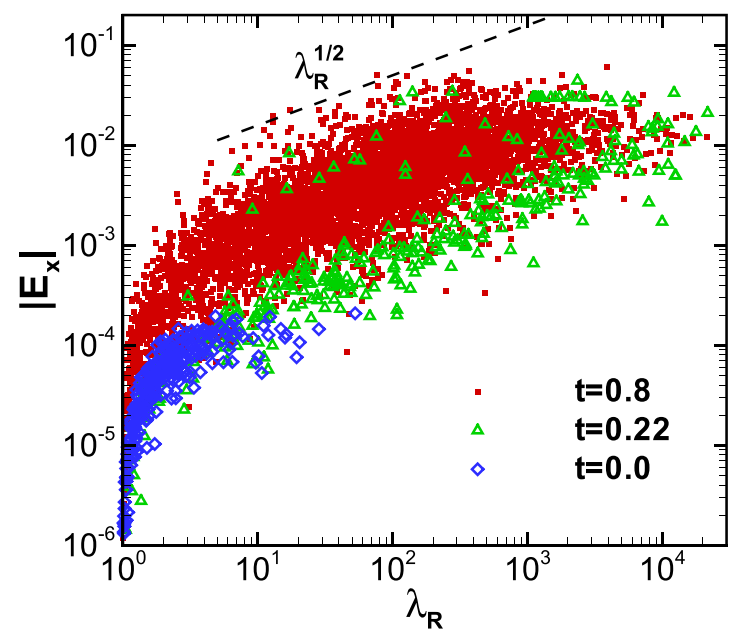

FIG. 10. The relation between the reconnection rate (the electric field at the $\mathrm{X}$-point) and the geometry of the reconnection region (the ratio of the eigenvalues) at different times of Run 8. The presence of a power-law fit (black dashed line) demonstrates that there is a relation between the reconnection rate and the geometry of the diffusion region. 

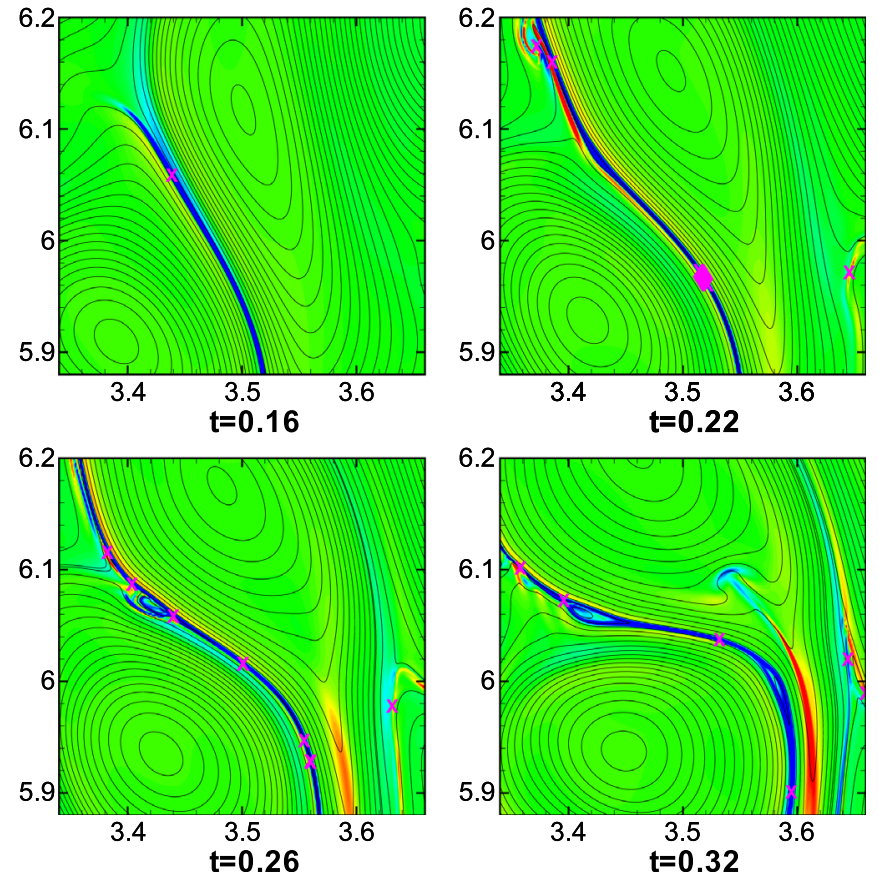

FIG. 11. Field-lines with X-points in a subregion at different times for Run 8 , which shows an example of generation of X-points. The color contour shows the current density $j$.
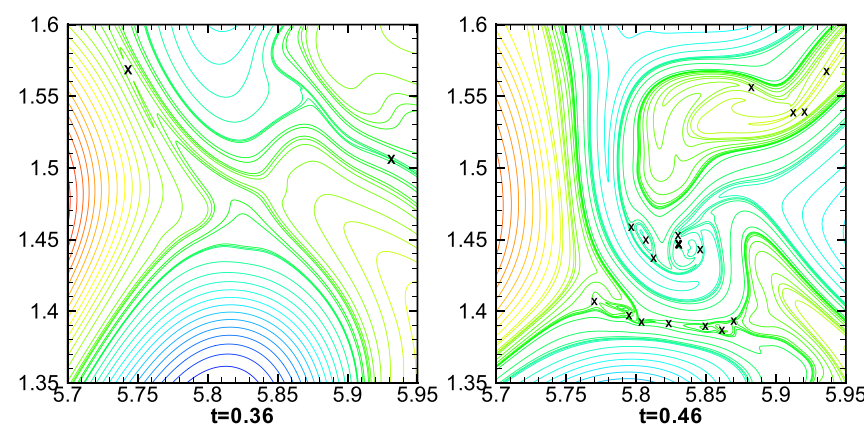

FIG. 12. Field-lines with X-points in a subregion at different times for Run 8 , showing an example of generation of X-points at later times.

has progressed-the region of interaction has become highly distorted, and the number of X-points has grown to at least 18. Evidently, regions of strong nonlinear interaction can tend to generate large numbers of emerging X-points.

\section{DISCUSSION AND CONCLUSIONS}

This paper has examined the production of new $\mathrm{X}$-points or secondary islands in two dimensional incompressible MHD turbulence. The production of new islands, or Opoints, and the production of new X-points are strictly related by the mathematical equality in periodic geometry of $\mathrm{X}$-point and O-point populations of any smooth surface such as the magnetic potential function. Of course, the ease of classification of critical points in 2D does not diminish the care needed to accurately determine their number and positions.

For fixed initial data and Reynolds number, we find that when well resolved, the number of X-points may increase or decrease with time in accordance with the development of the cascade. However, lack of adequate numerical resolution can easily increase the number of detected X-points, thus producing nonphysical results. Generally speaking, one requires high spatial resolution, to at least three times the Kolmogorov dissipation wavenumber, when using the pseudo-spectral approach that we have employed. Even with this high resolution in the computed dynamics, we further extend the resolution in the analysis phase by making use of a larger $\boldsymbol{k}$-space and zero padding, this providing a trigonometric interpolation of a smooth signal onto a finer grid. In this way, we ensure the accuracy of the topological characteristics that we discuss based on numerical experiments.

A general conclusion that we can draw is that high Reynolds number turbulence has the capacity to produce many more X-points starting from a band-limited initial condition. This number increases with Reynolds number and in time as the cascade expands in wavenumber to fill the accessible phase space. We find empirical support for three specific conclusions: (1) The density of "new" X-points (per unit square correlation length) increases as (Reynolds number $)^{3 / 2}$, which is readily understood as a consequence of a strong cascade; (2) the distribution of reconnection rates is found to have an approximately exponential distribution over a wide range of values; and (3) the rms reconnection rate decreases with increasing Reynolds number with no precise scaling found, although a simple argument suggests scaling as $R e_{m}^{-1 / 2}$.

We should note that the proliferation of plasmoids and $\mathrm{X}$-points, as we have described, leads to a complex dynamical situation in which reconnection rates at individual X-points might, on average, become slower, while macroscopic rates of magnetic flux transfer, being dependent on the net interaction among all sites, might remain rapid. Therefore, the proliferation of plasmoids may change the face of turbulence effects on reconnection, while maintaining the general conclusion that turbulence enhances the (macroscopically observed) rate of reconnection. The precise rules for composing microscopic rates into macroscopic rates remain a topic of discussion the literature; see, e.g., Refs. 11, 13, and 14.

The above conclusions apply to a 2D turbulent state. A similar increase of small secondary islands has been examined numerically employing laminar initial data, and the ensuing dynamics has been characterized as a "plasmoid instability."11-13,34 The physics seems to us to be essentially the same, noting, however, that no linear instability stage is required for the effect we have described, given the system we consider is never in an equilibrium. On the other hand, it is clear that one could construct a linear version of the process we have described, by artificially freezing certain Fourier modes that contain large amounts of energy, and considering the linear problem that certain of the less energetic modes follow in time. At the present time, we see little advantage in actually constructing and computing this linear system. Indeed, examining the numerical simulations that report plasmoid instability, ${ }^{1-13,34}$ it seems to us likely that the reported states with many plasmoids are already in a strongly nonlinear regime.

Our general conclusion is that the growth of the number of $\mathrm{X}$-points and secondary islands is a process intimately 
associated with the development of the cascade in turbulence. As such it is an effect that is more pronounced at higher Reynolds number, and which requires increasingly demanding spatial resolution as Reynolds number is increased. In fact, the required resolution for accurate determination of the number of X-points may be greater than some researchers might anticipate, and in this regard we urge caution to avoid numerical artifacts (see also Ref. 28). We suspect that a similar proliferation of critical points occurs in three dimensions, where the possibilities for topological complexity are still greater than in the simpler case considered here. 4

\section{ACKNOWLEDGMENTS}

This research was supported in part by the NSF Solar Terrestrial Program Grant No. AGS-1063439 and SHINE Grant No. AGS-1156094, NASA Heliophysics Theory Program, NNX11AJ44G, The Magnetospheric Multiscale Theory and modeling project, the Solar Probe Plus ISIS project, POR Calabria FSE-2007/2013, and by Marie Curie Project FP7 PIRSES-2010-269297 - (Turboplasmas).

${ }^{1}$ D. Biskamp, Phys. Fluids 29, 1520 (1986).

${ }^{2}$ W. H. Matthaeus and S. Lamkin, Phys. Fluids 29, 2513 (1986).

${ }^{3}$ V. Carbone, P. Veltri, and A. Mangeney, Phys. Fluids A 2, 1487 (1990).

${ }^{4}$ E. R. Priest and D. I. Pontin, Phys. Plasmas 16, 122101 (2009).

${ }^{5}$ E. G. Zweibel and M. Yamada, Annu. Rev. Astron. Astrophys. 47, 291 (2009).

${ }^{6}$ J. V. Shebalin, W. H. Matthaeus, and D. C. Montgomery, J. Plasma Phys. 29, 525 (1983).

${ }^{7}$ S. Oughton, E. R. Priest, and W. H. Matthaeus, J. Fluid Mech. 280, 95 (1994).

${ }^{8}$ J. W. Bieber, W. Wanner, and W. H. Matthaeus, J. Geophys. Res. 101, 2511, doi:10.1029/95JA02588 (1996).

${ }^{9}$ W. H. Matthaeus, J. J. Ambrosiano, and M. L. Goldstein, Phys. Rev. Lett. 53, 1449 (1984).

${ }^{10}$ W. H. Matthaeus and S. Lamkin, Phys. Fluids 28, 303 (1985).

${ }^{11}$ N. F. Loureiro et al., Phys. Plasmas 14, 100703 (2007).
${ }^{12}$ A. Bhjattacharjee et al., Phys. Plasmas 16, 112102 (2009).

${ }^{13}$ P. A. Cassak, M. A. Shay, and J. F. Drake, Phys. Plasmas 16, 120702 (2009).

${ }^{14}$ W. Daughton, V. Roytershteyn, B. J. Albright, H. Karimabadi, L. Yin, and K. J. Bowers, Phys. Rev. Lett. 103, 065004 (2009).

${ }^{15} \mathrm{G}$. Kowal, A. Lazarian, E. T. Vishniac, and K. Otmianowska-Mazur, Astrophys. J. 700, 63 (2009).

${ }^{16}$ S. Servidio, W. H. Matthaeus, M. A. Shay, P. A. Cassak, and P. Dmitruk, Phys. Rev. Lett. 102, 115003 (2009).

${ }^{17}$ S. Servidio, W. H. Matthaeus, M. A. Shay, P. Dmitruk, P. A. Cassak, and M. Wan, Phys. Plasmas 17, 032315 (2010).

${ }^{18}$ S. Servidio, P. Dmitruk, A. Greco, M. Wan, S. Donato, P. A. Cassak, M. A. Shay, V. Carbone, and W. H. Matthaeus, Nonlinear Processes Geophys. 18, 675 (2011).

${ }^{19}$ A. Lazarian, G. L. Eyink, and E. T. Vishniac, Phys. Plasmas 19, 012105 (2012).

${ }^{20}$ Z. B. Guo, P. H. Diamond, and X. G. Wang, Astrophys. J. 757, 173 (2012).

${ }^{21}$ B. U. O. Sonnerup, "Magnetic Field Reconnection," in Solar System Plasma Physics, edited by L. J. Lanzerotti, C. F. Kennel, and E. N. Parker (North-Holland, 1979), pp. 45-108.

${ }^{22}$ B. U. O. Sonnerup, in Solar Terrestrial Physics-Present and Future, edited by D. M. Butler and K. Papadopoulos, NASA Reference Publication 1120 (NASA, 1984), Chap. I.

${ }^{23}$ S. Servidio, F. Valentini, F. Califano, and P. Veltri, Phys. Rev. Lett. 108, 045001 (2012).

${ }^{24}$ H. Karimabadi, V. Roytershteyn, M. Wan, W. H. Matthaeus, W. Daughton et al., Phys. Plasmas 20, 012303 (2013).

${ }^{25}$ C. Canuto, M. Y. Hussaini, A. Quarteroni, T. A. Zang, Spectral Methods in Fluid Mechanics (Springer-Verlag, New York, 1988).

${ }^{26}$ G. S. Patterson and S. A. Orszag, Phys. Fluids 14, 2538 (1971).

${ }^{27}$ S. Servidio, W. H. Matthaeus, and P. Dmitruk, Phys. Rev. Lett. 100, 095005 (2008).

${ }^{28}$ M. Wan, S. Oughton, S. Servidio, and W. H. Matthaeus, Phys. Plasmas 17, 082308 (2010).

${ }^{29}$ A. S. Monin and A. M. Yaglom, Statistical Fluid Mechanics (MIT Press, Cambridge, MA, 1975), Vol. 2.

${ }^{30}$ P. D. Mininni and A. Pouquet, Phys. Rev. E 80, 025401 (2009).

${ }^{31}$ D. Ruffolo, W. H. Matthaeus, and P. Chuychai, Astrophys. J. 614, 420 (2004).

${ }^{32}$ W. H. Matthaeus, J. W. Bieber, D. Ruffolo, P. Chuychai, and J. Minnie, Astrophys. J. 667, 956 (2007).

${ }^{33}$ M. Wan, S. Oughton, S. Servidio, and W. H. Matthaeus, Phys. Plasmas 16, 080703 (2009)

${ }^{34}$ W. Daughton, J. Scudder, and H. Karimabadi, Phys. Plasmas 13, 072101 (2006). 